\title{
Teratocarcinosarcoma rinosinusal: Reporte de un caso
}

\section{Rhinosinusal teratocarcinosarcoma: A case report}

Claudia González G , Rodrigo Iñiguez S¹, Carolina Aranís Jiménez¹, Adriana Castiblanco Galvis².

\section{RESUMEN}

Teratocarcinosarcoma rinosinusal es una neoplasia maligna infrecuente y rara, que combina elementos de teratoma y carcinosarcoma. Se compone de epitelio benigno o maligno (fibroblastos), mesénquima (cartílago, hueso o músculo liso) y elementos neurales. Hasta el año 2008 se han reportado un total de 63 casos de ubicación rinosinusal.

Presentamos el caso de un teratocarcinosarcoma de fosa nasal, en un hombre de 67 años de edad, que consultó por obstrucción nasal rápidamente progresiva. La lesión se resecó completamente por abordaje endoscópico. 1 diagnóstico fue confirmado por histopatología e inmunohistoquímica. Se realizó tratamiento complementario con radioterapia, sin evidencia de recidiva al año después de la cirugía

Palabras clave: Teratocarcinosarcoma, tumor rinosinusal.

\begin{abstract}
Rhinosinusal teratocarcinosarcoma is a rare malignant neoplasm, which combines teratoma and carcinoma elements. It is composed of benign or malignant epithelium (fibroblasts), mesenchymatic (cartilage, bone or smooth muscle) and neural elements. Until 2008, a total of 63 cases of rhinosinusal location have been reported.

We present the case of a nasal cavity teratocarcinosarcoma in a 67 year old man that presented with rapidly progressing nasal obstruction. The lesion was completely resected by endoscopic approach. Diagnosis was confirmed by histological pathology and immunohistochemistry. Additional treatment by radiotherapy was administered, with no evidence of recurrences a year after surgery.
\end{abstract}

Key words. Teratocarcinosarcoma, rhinosinusal tumor.

Médico Otorrinolaringólogo. Departamento de Otorrinolaringología. Pontificia Universidad Católica de Chile.

2 Médico Anátomo-Patólogo. Pontificia Universidad Católica de Chile. 


\section{INTRODUCCIÓN}

Teratocarcinosarcoma rinosinusal es una infrecuente y rara neoplasia maligna, que combina elementos de teratoma y carcinosarcoma'. Se compone de epitelio benigno 0 maligno, mesénquima (cartílago, hueso 0 músculo liso) y elementos neurales². También ha sido llamado teratoma maligno ${ }^{3-5}$ carcinoma teratoide ${ }^{6,7}$, blastoma $^{8}$ y teratocarcinosarcoma maligno ${ }^{9}$.

Los hallazgos clínicos más característicos son un tumor en la cavidad nasal, que compromete el seno maxilar ipsilateral 0 el seno etmoidal. Suele ser un hallazgo de las cirugías transesfenoidales por neurocirujanos ${ }^{10}$.

Heffner y cols $^{11}$ en 1984 describió por primera vez esta neoplasia, y en una serie de 20 pacientes reportó una mortalidad de $60 \%$ a los 3 años, con un promedio de sobrevida de 1,7 años. Produce una agresiva extensión local, y ocasionalmente metástasis a linfonodos regionales y pulmón a los 3 años del dignóstico.

Teratocarcinosarcoma se presenta casi exclusivamente en el tracto sinonasal, reportándose hasta abril del 2008 un total de 63 casos $^{12}$; en Chile, no se han reportado casos. Sólo 6 han sido descritos fuera del tracto sinonasal: 4 en la nasofaringe, 1 en la cavidad oral y otro en la pared posterior de la faringe $\mathrm{e}^{13-15,2}$.

A continuación, reportamos el caso de un paciente con un teratocarcinosarcoma del tracto sinonasal, que presentó como primer síntoma obstrucción nasal.

\section{CASO CLÍnICO}

Paciente género masculino, de 67 años, con historia de varios años de evolución de obstrucción nasal izquierda. Cuatro meses previo a la consulta

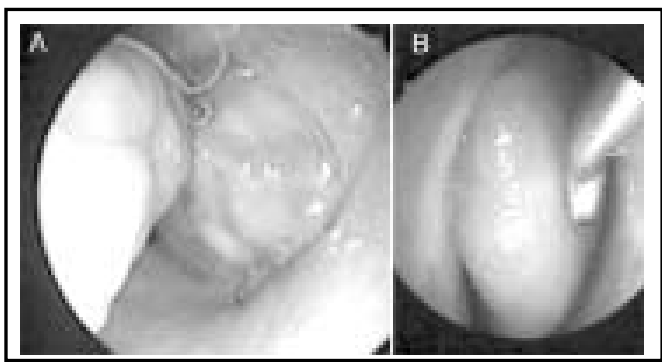

Figura 1. Endoscopía nasal. A. Fosa nasal derecha: coana completamente ocupada por masa tumoral. B. Fosa nasal izquierda: masa tumoral de aspecto similar al cornete medio que ocupa completamente la fosa nasal. comienza con obstrucción nasal progresiva a derecha, rinorrea serosa, descarga posterior y respiración bucal, asociado a hipoacusia bilateral.

Al examen nasal se encontró desviación septal oclusiva a izquierda, que al ser sobrepasada permitía visualizar una masa de consistencia firme de aspecto similar al cornete medio, pero que ocupaba completamente la fosa nasal izquierda. La fosa nasal derecha se observaba permeable, sin embargo, hacia posterior se encontró una masa tumoral que ocupaba completamente la coana (Figura 1). El resto del examen fue normal.

Se solicitó una tomografía axial computada (TAC) de cavidades paranasales que mostró velamiento pansinusal izquierdo, gran desviación anterior a izquierda del tabique nasal y presencia de una masa tumoral en fosa nasal izquierda que se extiende a rinofaringe y ocluye ambas coanas, compatible con el diagnóstico de papiloma invertido v/s pólipo antrocoanal (Figura 2).

Se realizó un abordaje endoscópico para la cirugía. Encontrándose un tumor nasal creciendo hacia rinofaringe, de consistencia muy firme, con un pedículo entre cornete medio y tabique nasal, sin invasión de estructuras adyacentes. Se resecó completamente, y se electrocauterizó la zona del pedículo a nivel de base de cráneo.

El estudio anátomo-patológico concluyó como diagnosticó teratocarcinosarcoma rinosinusal. Basándose en la presencia de células con diferenciación neural, estructuras glandulares con atipía y

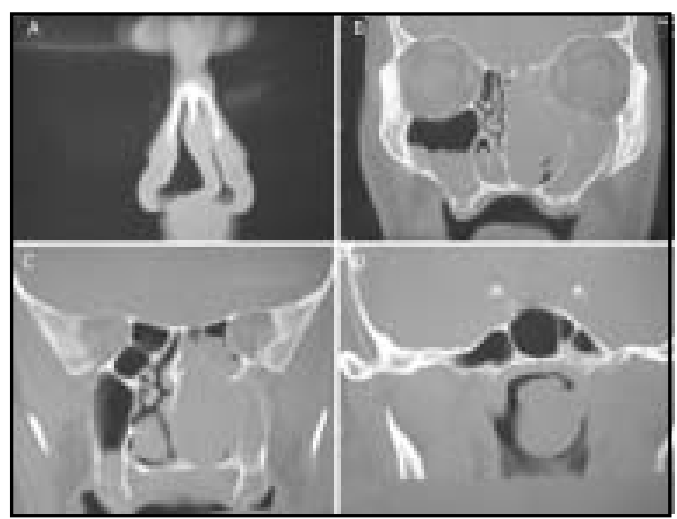

Figura 2. TAC de senos paranasales. Cortes coronales. A. Desviación septal anterior a izquierda. B. Masa tumoral que ocupa fosa nasal izquierda y desplaza hacia lateral la pared medial del seno maxilar. Velamiento maxilar ipsilateral. C. Masa tumoral se extiende hacia zona posterior de fosa nasal. D. Masa tumoral a nivel de coanas y rinofaringe. 


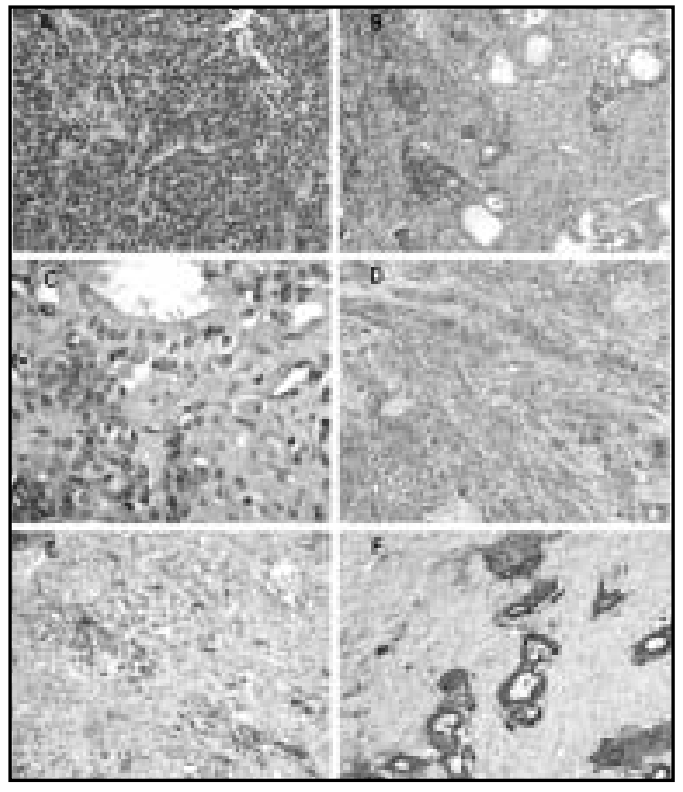

Figura 3. Histopatología e inmunohistoquímica. A. Áreas con células pequeñas de núcleos hipercromáticos dispuestas en una matriz fibrilar con diferenciación neural. H-E (hematoxilinaeosina) 10X. B y C. Estructuras glandulares con atipía citológica, células primitivas de tipo blastoma que esbozan rosetas (células dispuestas en torno a un centro ópticamente vacío, que no corresponde a un vaso) y un componente sarcomatoso inmaduro con células de aspecto rabdoide (a la izquierda). H-E 10X. D. Inmunohistoquímica para CD34 positiva en el componente sarcomatoso. (Proteína transmembrana inicialmente detectada en células hematopoyéticas de la línea mieloide y endotelio vascular. Útil en la diferenciación de tumores fusocelulares como sarcomas y lipomas). E. Desmina fue positiva en las células de aspecto rabdoide. (Proteína estructural de las miofibrillas). F. Positividad inmunohistoquímica para citoqueratinas totales (AE1/ AE3) destaca el componente epitelial maligno. (Componente del citoesqueleto de células epiteliales).

células de aspecto sarcomatoso de tipo rabdoide. El estudio con inmunohistoquímica fue positivo para CD34 en el componente sarcomatoso, positivo para desmina en las células de aspecto rabdoide y positivo para citoqueratinas totales (AE1/AE3) en el componente epitelial maligno (Figura 3).
En vista de estos hallazgos, se explicó al paciente la necesidad de una segunda intervención quirúrgica para ampliar el margen libre de tumor en la zona de inserción del teratocarcinosarcoma a nivel de base de cráneo, cirugía que el paciente rechazó.

Se etapificó mediante TAC de tórax y pelvis, los que resultaron negativos para diseminación tumoral. El paciente recibió tratamiento complementario con radioterapia en otro centro asistencial. Se realizó seguimiento endoscópico cada 4 meses, no encontrando evidencias macroscópicas de recidiva tumoral a los 12 meses posresección quirúrgica (Figura 4). Se realizó TAC de cavidades paranasales control encontrando sólo cambios posquirúrgicos, sin evidencia de recidiva. Hasta mayo 2008 el paciente se encontraba vivo.

\section{DISCUSIÓN}

Teratocarcinosarcoma es una neoplasia maligna histológicamente heterogénea, que muestra hallazgos combinados de un teratoma maligno y un carcinosarcoma, generalmente muy agresiva, se presenta casi únicamente en cavidad nasal o senos paranasales y es muy infrecuente. Hasta 2008 se han reportado 63 casos, lo que reafirma lo inusual de esta patología.

El término teratocarcinosarcoma es preferible, porque estas lesiones no se originan aparentemente de células germinales y son histológicamente diferentes de los teratomas malignos que se observan en otras partes del cuerpo.

Afecta a personas entre los 18 y 79 años de edad (promedio 51,7 años), predominantemente a hombres (5:1). La forma de presentación más frecuente es obstrucción nasal y epistaxis ${ }^{16}$.

El diagnóstico histológico se basa en la combinación de elementos epiteliales malignos y dos 0 más componentes mesenquimatosos malignos, como fibroblastos, cartílago, hueso y músculo liso. Cuando

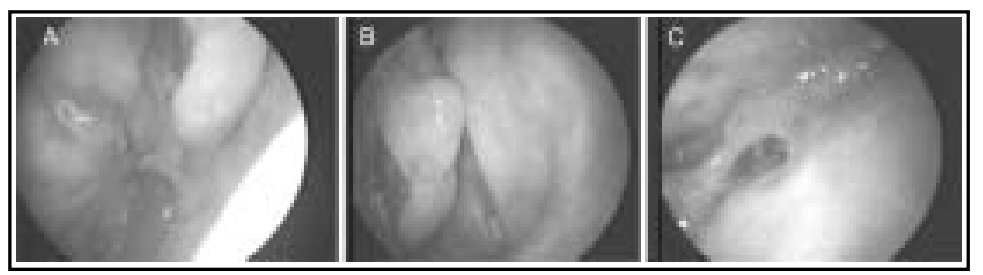

Figura 4. Control endoscópico al año. A. Fosa nasal derecha. B. Fosa nasal izquierda. C. Base de cráneo. Zona de inserción del tumor, sin evidencia de recidiva. 
sólo se encuentra un componente, recibe el nombre de carcinosarcoma ${ }^{17}$. En nuestro paciente, estuvieron presentes elementos epiteliales malignos, neurales, sarcomatosos, glandulares y blastomas, demostrados por el estudio inmunohistoquímico.

Este tumor muestra un rápido crecimiento y destrucción local extensa, con un infortunado pronóstico. Sin embargo, se han reportado 13 casos en los cuales no se evidenció recurrencia después de la resección, siendo el período de seguimiento más extenso de nueve años. La mayoría de éstos tuvo lesiones polipoídeas, y no mostraron invasión local macroscópica ni microscópica ${ }^{18}$, al ser tratados mediante cirugía y radioterapia.

Se describe como el tratamiento más efectivo a la resección quirúrgica más radioterapia. Debido al pequeño número de casos, la efectividad de la quimioterapia no ha podido ser evaluada, sin embargo, como terapia coadjuvante puede mejorar la recurrencia o lesiones metastásicas².

Según lo descrito, el manejo de nuestro paciente fue adecuado, con una evolución favorable y esperable según la evidencia. Al año de seguimiento se encontraba sin recidiva ni metástasis, lo que se ajusta a los reportes mencionados.

\section{BIBLIOGRAFÍA}

1. TaKasaki $K$, SaKiHama N, TaKahashi H. A case with sinonasal teratocarcinosarcoma in the nasal cavity and ethmoid sinus. Eur Arch Aorhinolaryngol 2006; 263(6): 586-91.

2. Carrizo F, Pineda-Daboin K, Galvao A, Luna M. Pharyngeal teratocarcinosarcoma: review of the literature and report of two cases. Annals of Diagnostic Pathology 2006; 10: 339-42.

3. Dicke TE, Gates GA. Malignant teratoma of the paranasal sinuses. Report of a case. Arch Otolaryngol 1970; 91: 391-4.

4. Авт AB, Toker C. Malignant teratoma of the paranasal sinuses. Arch Pathol 1970; 90: 176-80.

5. Patchefsky $A$, Sundmaker $W$, Marden Pa. Malignant teratoma of the ethmoid sinus. Report of a case. Cancer 1968; 21: 714-21.
6. Shanmugaratnam K, Kunaratnam N, Chia KB, Chiang GS, SinNiaH R. Teratoid carcinosarcoma of the paranasal sinuses. Pathology 1983; 15: 413-9.

7. Christensen EC, Fu YS, Wilson LJ, Hoover LA. Teratoid carcinosarcoma of the nasal and paranasal cavities: a case report. Am JRhinol 1992; 6: 169-72.

8. Sharma $H$, Abdullah JM, Othman NH, et al. Teratocarcinosarcoma of the nasal cavity and ethmoid. J Laryngol Ool 1998; 112: 682-6.

9. Shemen L, Galantich P, Murali R. Malignant teratocarcinosarcoma of the sphenoid sinus. Colaryngol Head Neck Surg 1995; 112: 496-500.

10. Terasaka S, Medary M. Prolonged survival in a patient with sinonasal teratocarcinosarcoma with cranial extension. Case report. J Neurosurg 1998; 88: 753-6.

11. HefFNeR DK, Hyams VJ. Teratocarcinosarcoma (malignant teratoma?) of the nasal cavity and paranasal sinuses. A clinicopathological study of 20 cases. Cancer 1984; 53: 2140-54.

12. Salem F, Rosenblum MK, Jhanwar SC, Kancherla $P$, Ghossein RA, Carlson DL. Teratocarcinosarcoma of the nasal cavity and paranasal sinuses: report of 3 cases with assessment for chromosome 12p status. Hum Pathol 2008; 39(4): 605-9.

13. Meinecke R, Bauer F, Skouras J, et al. Blastomatous tumors of the respiratory tract. Cancer 1974; 38 : 63-9.

14. Rotenberg B, El-Hakim H, Lodha A, et al. Nasopharyngeal teratocarcinosarcoma. Int $\mathrm{J}$ Pediatr Otorhinolaryngol 2002; 62: 159-64.

15. Pal SA, Naresh KN, Masih K, Ramarao C, Borges aM. Teratocarcinosarcoma of the paranasal sinuses: a clinicopathologic and immunohistochemical study. Hum Pathol 1998; 29: 718-22.

16. Hideko Endo, Takanori Hirose. Case Report: Sinonasal teratocarcinosarcoma. Pathology Internacional 2001; 51: 107-12.

17. Shindo ML, Stanley RB JR, Kiyabu MT. Carcinosarcoma of the nasal cavity and paranasal sinuses. Head Neck 1990; 12: 516-9.

18. Nitsche M, Hermann RM, Chistiansen H, et al. Rationale for individualized therapy in sinonasal teratocarcinosarcoma: Case report. Onkologie 2005; 28: 653-6.

Dirección: Dra. Claudia González G.

Departamento de Otorrinolaringología, Pontificia Universidad Católica de Chile

Marcoleta № 352, 2ำ piso. Teléfono: 3543484

E mail: cgonzale@med.puc.cl 